
2 Research Square
Preprints are preliminary reports that have not undergone peer review.
They should not be considered conclusive, used to inform clinical practice, or referenced by the media as validated information.

\title{
Effects of nano and ionized silicon on physiological and biochemical characteristics of potato (Solanum tuberosum L.)
}

\author{
Bijan Saadatian \\ Ferdowsi University of Mashhad \\ Mohamad Kafi \\ Ferdowsi University of Mashhad \\ Mohamad Banayan aval \\ Ferdowsi University of Mashhad \\ Hossein Hammami ( $\nabla$ homamihossein@gmail.com ) \\ University of Birjand
}

\section{Research Article}

Keywords: Antioxidant capacity, Chlorophyll content, Nano-Si, Soilless culture system

Posted Date: August 14th, 2021

DOI: https://doi.org/10.21203/rs.3.rs-781426/v1

License: () (1) This work is licensed under a Creative Commons Attribution 4.0 International License. Read Full License 


\section{Abstract}

The role of protecting and structure stabilizing effects of silicon (Si) has been demonstrated on different plant species. Still it has not been used in potato seed production under a soilless culture system. Furthermore, particle size is very important in particle adhesion and interactions with biological reactions. Therefore, the use of nano-Si particles may be more efficient than ionized -Si. For this purpose, a greenhouse experiment under a soilless culture system was performed as a randomized complete block design (RCBD) arranged in a factorial with three replications. In this study, Si concentration (distilled water

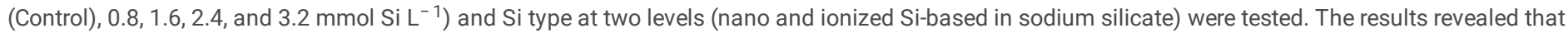
foliar application of Si significantly improved the net photosynthesis rate, water use efficiency, mesophyll conductance, Chl $a, \mathrm{Chl} b$, carotenoids, Chl $a / b$ ratio, DPPH radical scavenging, total phenol, mean weight mini-tuber, and yield, whereas transpiration rate in Si-treated plants decreased. Moreover, the greatest positive influence of Si was observed at $3.2 \mathrm{mmol} \mathrm{L}^{-1}$. The effect of Nano-Si was greater than ionized-Si at all Si concentrations. The results revealed that improved biochemical and photosynthetic characteristics of potato plantlet under Nano-Si treatments compared to ionized-Si treatments. However, these relations were not significant under ionized treatment. This study indicated that the application of Si (nano and ionized) for potato growing and mini-tuber production has positive effects. Generally, under soilless culture system, Nano-Si have higher efficiency than ionized-Si in mini-tuber production.

\section{Introduction}

Potato (Solanum tuberosum L.) is the world's fourth most important food crop after wheat, rice, and maize. In 2019, the potato was planted on 17340986 and 104192 ha with attainable yields of 21.3619 and 33.4324 ton ha ${ }^{-1}$ in the world and Iran, respectively [1]. It is widely used in the food industry such as chips and starch. Moreover, it is operated as biofuel and natural polymers [2]. The maintenance of potato production systems is closely dependent on a sufficient and continuous supply of high-quality seed tubers [3]. To produce healthy potato seeds from pathogens and especially viruses, potato plantlets are propagated from meristem culture and transferred to the greenhouse [3, 4].

Silicon ( $\mathrm{Si}$ ) is the second most (27.7\%) abundant mineral element in the earth crust and mineral substrate for most of the plants. Many researchers have shown that Si supply improved growth and development of crop plants under normal [5,6,7] and stressful conditions such as salinity [8], drought [9] and toxic heavy metals [10,11,12]. Therefore, it can be considered as a quasi-essential element [13]. Asmar et al. (2013) [14] reported that using silicon salts in micropropagation of banana (Musa spp.) plantlets can improve photosynthesis rate and chlorophyll content. It has been found that Si has beneficial effects in the antioxidant capacity $[15,16]$ and non-enzymatic reactive oxygen species (ROS) scavenger such as phenolic compounds [17, 18]. Zhang et al. (2017) [19] found that application of silicate fertilizer increased fruit yield, cluster weight, berry weight, and berry size of grape (Vitis vinifera L.) cultivars. Despite the positive results observed in many prior studies, Si has not been included in the formulation of nutrient solutions. However, not all plants are unable to absorb actively the silicon through the root except plants in Poaceae and Cyperaceae families [13]. Therefore, foliar application of this element may be an effective strategy for Si plant nutrition $[11,16]$.

Recently, nanotechnology has revolutionized the world with tremendous developments in many fields of science [20, 21]. Engineered nanoparticles (ENPs), with sizes smaller than $100 \mathrm{~nm}$ in at least one dimension, have received a lot of attention [12, 16]. Haghighi et al. (2012) [21] reported that the application of $1 \mathrm{mM}$ nano-Si led to improved germination rate, root length and dry weight of tomato (Lycopersicum esculentum) seedlings. Mahdavi et al. (2016) [22] also mentioned that application of nano Si enhanced chlorophyll index and concentration of essential elements such as N, P, Zn, and Cu in Perennial ryegrass (Lolium perenne) shoot under water stress. Although, Haghighi and Pessarakli (2013) [8] indicated that nano and bulk Si particles improved photosynthesis, mesophyll conductance, and plant water use efficiency under saline stress conditions in cherry tomato (Solanum lycopersicum L.), but they did not found a significant differences between nano and bulk Si application. Wang et al. (2014) [16] also found that the foliar application of nano-Si, prepared by using sodium silicate, alleviated cadmium (Cd) toxicity in seedling rice (Oryza sativa L.) by decreasing Cd accumulation and malondialdehyde (MDA) and increasing essential element contents of $\mathrm{Mg}$, Fe, and $\mathrm{Zn}$ and antioxidant capacity. Similar results are reported the interaction between Si nanoparticles and chromium stress in pea (Pisum sativum L.) seedlings [12].

Considering that in soilless greenhouse production of seed potato, the soilless substrate as growth media, and nutrition solutions do not have silicon sources [4]. The application of silicon has not been investigated yet. Therefore, this study was conducted to assess Si supplementation effects through the foliar application on potato transplanted plantlets, potato growth, photosynthetic parameters, biochemical characteristics, and mini-tuber yield. It is also tried to find that the effects of Si particle size on growth and biochemical characteristics.

\section{Materials And Methods}

\subsection{Experiment}

This greenhouse experiment was conducted at the College of Agricultural, Ferdowsi University of Mashhad (FUM), Khorasan Razavi Province, Mashhad (36 $\left.27^{\prime} \mathrm{N}, 59^{\circ} 63^{\prime} \mathrm{E}\right)$, Iran, in 2015. The experiment was conducted as a randomized complete block design (RCBD) with a factorial arrangement in three replications (experimental unit has five plantlets). Foliar application of silicon (Si) concentrations (distilled water (control), 0.8, 1.6, 2.4, and 3.2 mmol Si L-1) and the silicon ( $\mathrm{Si}$ ) particle size (nano and ionized) were considered as experimental factors, respectively. Treatments were sprayed using an overhead trolley sprayer (Matabi 121030 Super Agro 20 L sprayer; Agratech Services-Crop Spraying Equipment, Rossendale, UK) at two growth stages: stolon (21 days after transplanting) and tuber (32 days after transplanting) initiation stages. At each time of application, $25 \mathrm{ml}$ of Si solution was sprayed for each plantlet. This experiment was repeated twice under the same condition.

\subsection{Synthesis of silicon nanoparticles}


Nanoparticles of sodium silicate produced in Central Lab of Ferdowsi University of Mashhad. At the elementary phase, $50 \mathrm{~g}$ of $\mathrm{Na}_{2} \mathrm{SiO}_{3}(\mathrm{Sigma} \mathrm{Aldrich}$ code number product: $307815,99.9 \%$ purity, white color, and granule) were heated in an oven at $75^{\circ} \mathrm{C}$ for $5 \mathrm{~h}$ and was ground in a mixer mill (MM 400 , Retsch, Germany) by $1680 \mathrm{rpm}$ for 50 min with 1:10 of ratio sample to balls (w/w). Finally, bulk and milled particles size were measured with Particle Size Analyzer (PSA) (VASCO3, Cordouan Technologies, France, rangs of measurement $6 \mu \mathrm{m}$ to $1 \mathrm{~nm}$ ) and Scanning Electron Microscope (SEM, model 1450VP, LEO, Germany, magnification ranging from $20 x$ to approximately $300000 x$, and $2 \mathrm{~nm}$ resolution). The average diameter of milled particles was $68 \mathrm{~nm}$ (Figs. 1 and 2 ).

Please insert Figs. 1 and 2 near hear

\subsection{Preparation of nano and ionized solutions}

For preparation of each concentration level (nano or ionized), Citogait (a nonionic surfactant, 100\% alkyl aryl polyglycol ether, Zarnegaran Pars, Iran) was added at $0.2 \%(\mathrm{v} / \mathrm{v})$ to $500 \mathrm{~mL}$ of particle solution and its $\mathrm{pH}$ was adjusted to $5 \pm 0.1$ with $0.01 \mathrm{~N} \mathrm{HCl}$. Then, the solutions shacked at $250 \mathrm{rpm}$ in darkness at $20^{\circ} \mathrm{C}$ for $2 \mathrm{~h}$.

\subsection{Providing plantlets}

To prepare the potato plantlets (Agria cv.), stem segments free from pathogens (approximately $15 \mathrm{~mm}$ length with one leaf node) were grown in vitro by using

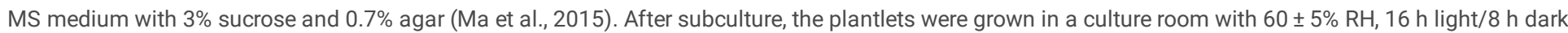
period (approximately $400 \mu \mathrm{mol}$ photons $\mathrm{m}^{-2} \mathrm{~s}^{-1}$ ) at $24 \pm 2^{\circ} \mathrm{C}$ for 21 days. Afterward, each uniformed plantlet was gently washed with distilled water to remove agar and transplanted into a plastic pot (30 and $12 \mathrm{~cm}$ depth and diameter, respectively) filled with a 1:1:1 perlite, cocopite, and sand media. Fifty percent of growth medium was added in transplanting time and the rest was added after four weeks.

\subsection{Experimental conditions}

During the experiment, the mean of irradiance was approximately $1000 \mu \mathrm{mol}$ photon $\mathrm{m}^{-2} \mathrm{~s}^{-1}$ with $14 \mathrm{~h}$ light/10 h dark photoperiod, and the maximum and minimum air temperatures were $26^{\circ} \mathrm{C}$ and $18^{\circ} \mathrm{C}$, respectively. Mean relative humidity was also $40 \pm 10 \%$. Irrigation was applied equally (200 mL per plantlet) twice a week. The plantlets were nourished with $100 \mathrm{~mL}$ of complete Hoagland nutrient solution [23] once a week. The pH was measured with portable equipment and adjusted for $5.5 \pm 0.1$ using $\mathrm{HCl}$ and $\mathrm{NaOH} 2 \mathrm{~N}$ solutions. To prevent the accumulation of salt every 15 days, $1200 \mathrm{~mL}$ of distilled water was added to each pot to wash the medium.

\subsection{Gas exchange parameters}

One week after second silicon application spraying, leaf gas exchange parameters including net photosynthetic rate $\left(\mathrm{Pn}, \mu \mathrm{mol} \mathrm{CO} \mathrm{m}^{-2} \mathrm{~s}^{-1}\right)$, substomatal $\mathrm{CO}_{2}$ concentration $\left(\mathrm{Ci}, \mathrm{CO}_{2} \mathrm{ppm}\right)$, transpiration rate $\left(\mathrm{Tr}, \mathrm{mmol} \mathrm{m} \mathrm{m}^{-2} \mathrm{~s}^{-1}\right)$ and stomatal conductance (Gs, $\mathrm{mmol} \mathrm{m}^{-2} \mathrm{~s}^{-1}$ ) were recorded from 10:00 to $11: 00$ a.m. in the clear and sunny sky by using a compact portable photosynthesis measurement system (LCi leaf chamber Analysis, ADC Bio Scientific Ltd. Hoddsdon, UK). Mesophyll conductance ( $\mathrm{MC}, \mathrm{mmol} \mathrm{CO}_{2} \mathrm{~m}^{-2} \mathrm{~s}^{-1}$ ) was calculated as the net photosynthetic rate divided by the substomatal $\mathrm{CO}_{2}$ concentration. Water use efficiency (WUE, $\mu \mathrm{mol} \mathrm{CO} \mathrm{mmol}^{-1} \mathrm{H}_{2} \mathrm{O}$ ) was calculated as the net photosynthesis rate divided by the transpiration rate [8]. To assess these variables, an average of eight fully developed potato leaves was measured from each replication.

\subsection{Biochemical assay}

One week after the second Si application, chlorophyll (Chl), carotenoids (Cart), total phenol and DPPH radical-scavenging were measured from the second youngest fully developed leaves.

\subsubsection{Pigments}

Chlorophyll $a$ and $b$ and carotenoids contents were extracted based on Arnon (1949) [24] method. Samples of 100 mg fresh leaves were homogenized with $80 \%$ methanol at $4^{\circ} \mathrm{C}$ in a micro-tube and centrifuged at $3000 \mathrm{~g}$ for $15 \mathrm{~min}$ at $4^{\circ} \mathrm{C}$ and put in the refrigerator for $24 \mathrm{~h}$. Then, the upper extract absorbance was measured at 653,666 , and $470 \mathrm{~nm}$ with a UV/Vis spectrophotometer (JENEWAY, model 6305, UK). Chlorophyll content was calculated using the formula and expressed in $\mathrm{mg}$ per $\mathrm{g}$ fresh weight. The chlorophyll $a / b$ ratio was also calculated as described by Arnon (1949) [24].

\subsubsection{Total phenols}

The total phenol content was determined according to the Folin-Ciocalteu reagent method proposed by Singleton and Rossi (1965) [25] with slight modification. Briefly, the alcoholic extract $(20 \%, \mathrm{w} / \mathrm{v})$ was diluted with distilled water and Folin-Ciocalteau was added to the mixture. After five minutes, the sodium carbonate $(20 \%, \mathrm{w} / \mathrm{v})$ was added and the mixture was kept in the dark for $30 \mathrm{~min}$. The absorbance was measured at $765 \mathrm{~nm}$ (JENWAY, model 6305 , UK), and total phenols were expressed as $\mathrm{mg}$ of gallic acid per $\mathrm{g}$ fresh weight.

\subsubsection{DPPH radical scavenging}

The DPPH radical scavenging activity of leaf extracts was measured according to the modified Abe et al. (1998) [26] method. The alcoholic extract (20\%, w/v) was mixed with alcoholic DPPH solution $(8 \%, \mathrm{w} / \mathrm{v})$. The reaction mixture was shaken vigorously and left to stand in darkness for 30 min and absorbance was measured at $517 \mathrm{~nm}$ (JENWAY, model 6305, UK). DPPH radical scavenging was expressed as mg of ascorbic acid per $\mathrm{g}$ fresh weight.

\subsection{Harvest of mini-tuber}

The economic yield, including the mean mini-tuber weight and yield per plant was measured after 95 days of transplanting.

\subsection{Statistical analysis}


The study was arranged in a pooled two factorial experiments design with six replications. The data were analyzed with SAS 9.1 (SAS, Institute Inc., NC). Significant differences between the treatments were determined using Fisher's Least Significant Difference (FLSD) test at 0.05 probability level. Pearson correlation coefficient was also used to determine the correlation between traits.

\section{Results}

\subsection{Gas exchange parameters}

Silicon particle size $(\mathrm{P})$ significantly affected net photosynthetic rate (Pn), Mesophyll conductance (MC), and water use efficiency (WUE) in potato plantlets, but no significant effect was observed on transpiration rate ( $\mathrm{Tr}$ ) and substomatal $\mathrm{CO}_{2}$ concentration (Ci) (Table 1). Whereas, silicon concentration levels (C) showed a significant effect on all measured gas exchange parameters. A significant interaction between $\mathrm{P}$ and $\mathrm{C}$ was also observed on $\mathrm{Pn}, \mathrm{Ci}$, MC and WUE (Table 1).

Table 1

Analysis of variance for the effect of particle size and Si concentration levels on gas exchange parameters, pigment contents, biochemical traits, and tuber yi

\begin{tabular}{|c|c|c|c|c|c|c|c|c|c|c|c|c|c|}
\hline $\begin{array}{l}\text { Source of } \\
\text { variation }\end{array}$ & d.f & ${ }^{\text {apn }}$ & $\mathrm{Tr}$ & $\mathrm{Ci}$ & $M C$ & Gs & WUE & Chl a & Chl $b$ & $\begin{array}{l}\text { Chl } \\
a / b\end{array}$ & Cart & DPPH & $\begin{array}{l}\mathrm{T} \\
\mathrm{ph}\end{array}$ \\
\hline Replication & 5 & $\begin{array}{l}\text { b } 0.05 \\
\text { NS }\end{array}$ & $\begin{array}{l}0.04 \\
\text { NS }\end{array}$ & $2391^{*}$ & $\begin{array}{l}0.0000009 \\
\text { NS }\end{array}$ & $12.3^{*}$ & $\begin{array}{l}0.03 \\
\text { NS }\end{array}$ & $\begin{array}{l}0.004 \\
\text { NS }\end{array}$ & 0.0009 NS & $\begin{array}{l}0.024 \\
\text { NS }\end{array}$ & $\begin{array}{l}0.0001 \\
\text { NS }\end{array}$ & $\begin{array}{l}0.000015 \\
\text { NS }\end{array}$ & $0 . c$ \\
\hline $\begin{array}{l}\text { Si particle } \\
\text { size }(P)\end{array}$ & 1 & $0.74^{\star *}$ & $\begin{array}{l}0.05 \\
\text { NS }\end{array}$ & $1706^{N S}$ & $0.0000028^{*}$ & $276.7^{N S}$ & $0.24^{*}$ & $0.066^{\star \star \star}$ & $0.00090^{\star \star \star}$ & $\begin{array}{l}0.001 \\
\text { NS }\end{array}$ & $0.0006^{* *}$ & $\begin{array}{l}0.000003 \\
\text { NS }\end{array}$ & $\begin{array}{l}0 . C \\
\text { NS }\end{array}$ \\
\hline $\begin{array}{l}\text { Si } \\
\text { concentration } \\
\text { (C) }\end{array}$ & 4 & $2.6^{\star \star \star}$ & $1.76^{\star \star \star}$ & $15199^{\star \star \star}$ & $0.0000091^{\star \star \star}$ & $\begin{array}{l}234.1 \\
\text { NS }\end{array}$ & $4.61^{\star \star \star}$ & $0.039^{\star \star \star}$ & $0.00200^{\star *}$ & $0.138^{*}$ & $0.0004^{\star *}$ & $0.000206^{\star \star *}$ & $0 . c$ \\
\hline$P \times C$ & 4 & $0.2^{*}$ & $\begin{array}{l}0.05 \\
\text { NS }\end{array}$ & $5668^{\star \star \star}$ & $0.0000022^{\star}$ & $369^{N S}$ & $0.17^{*}$ & $0.009^{*}$ & $0.00114^{*}$ & $\begin{array}{l}0.008 \\
\text { NS }\end{array}$ & $\begin{array}{l}0.0001 \\
\text { NS }\end{array}$ & $0.000240^{\star \star *}$ & $0 . C$ \\
\hline CV (\%) & - & 7.1 & 11.3 & 7.3 & 9.3 & 8.2 & 13.1 & 6.8 & 6.5 & 8.8 & 7.3 & 15.2 & 11 \\
\hline
\end{tabular}

a Net photosynthetic rate (Pn), Transpiration rate (Tr), Substomatal $\mathrm{CO}_{2}$ concentration (Ci) Mesophyll conductance (MC), Gs (stomatal conductance), water u chlorophyll $a(\mathrm{Chl} a)$, chlorophyll $b(\mathrm{Chl} b)$, carotenoids (Cart), DPPH radical scavenging (DPPH), total phenol (T. phenol), mini-tuber weight (TW), mini-tuber yi

b NS: not significance at $P>0.05 ; *, * *, * \star *$ significance level at $P<0.05,0.01$ and 0.001 .

Please insert Table 1 near hear

Foliar application of nano-Si and ionized-Si improved the net photosynthetic rate, mesophyll conductance, and water use efficiency (Table 2). The increasing Si concentrations, increased net photosynthesis rate, but the different sizes of Si had no similar effects. Net photosynthesis rate was significantly increased

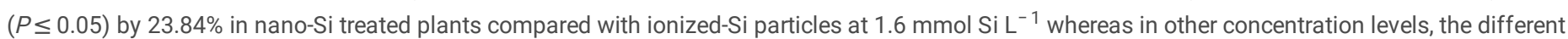

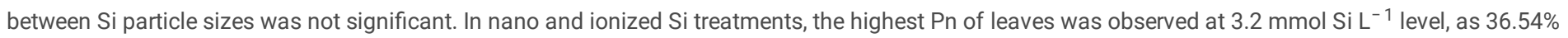
and 33.02\% increment compared with control, respectively (Table 2). Although the interaction between particle size and Si concentration level was not significant on leaf transpiration rate (Table 1), transpiration rate distinctively decreased by increasing Si concentration in tuberization stage, and all Si concentration levels, a significant difference with control was recorded $(P<0.05)$ (Table 3$)$. Furthermore, the highest value of transpiration rate was also obtained at $3.2 \mathrm{mmol} \mathrm{Si} \mathrm{L}^{-1}$ level with an increase of $38.06 \%$ compared with control (Table 4). 
Table 2

Interaction effects of the Si particle size $(\mathrm{P})$ and Si concentration levels $(\mathrm{C})$ on the net photosynthetic rate (Pn), substomatal CO2 concentration (Ci), mesophyll conductance (MC) and water use efficiency (WUE) of potato plantlet leaves at tuberization stage.

\begin{tabular}{|c|c|c|c|c|c|}
\hline \multicolumn{2}{|l|}{ Treatments } & \multicolumn{4}{|l|}{ Traits } \\
\hline $\mathbf{P}$ & C & Pn & $\mathrm{Ci}$ & MC & WUE \\
\hline & & $\left(\mu \mathrm{mol} \mathrm{CO} \mathrm{C} \mathrm{m}^{-2} \mathrm{~s}^{-1}\right)$ & $\left(\mathrm{CO}_{2} \mathrm{ppm}\right)$ & $\left(\mathrm{mmol} \mathrm{CO} \mathrm{Cm}^{-2} \mathrm{~s}^{-1}\right)$ & $\left(\mu \mathrm{mol} \mathrm{CO} 2 \mathrm{mmol}^{-1} \mathrm{H}_{2} \mathrm{O}\right)$ \\
\hline \multirow{5}{*}{$\begin{array}{l}\text { Nano-Si } \\
\left(\mathrm{mmol} \mathrm{L}^{-1}\right)\end{array}$} & Control & $3.12 \pm 0.25$ & $395 \pm 36$ & $7.98 \pm 1.14$ & $1.28 \pm 0.14$ \\
\hline & 0.8 & $3.36 \pm 0.21$ & $388 \pm 32$ & $8.69 \pm 0.91$ & $1.59 \pm 0.06$ \\
\hline & 1.6 & $3.48 \pm 0.22$ & $372 \pm 24$ & $9.40 \pm 1.11$ & $1.69 \pm 0.23$ \\
\hline & 2.4 & $3.78 \pm 0.42$ & $494 \pm 45$ & $7.70 \pm 0.87$ & $2.32 \pm 0.28$ \\
\hline & 3.2 & $4.26 \pm 0.14$ & $399 \pm 27$ & $10.69 \pm 0.57$ & $2.68 \pm 0.19$ \\
\hline \multirow[t]{5}{*}{ Ionized-Si $\left(\mathrm{mmol} \mathrm{L}^{-1}\right)$} & Control & $3.15 \pm 0.32$ & $395 \pm 41$ & $8.01 \pm 0.55$ & $1.29 \pm 0.20$ \\
\hline & 0.8 & $3.20 \pm 0.19$ & $398 \pm 27$ & $8.04 \pm 0.44$ & $1.39 \pm 0.19$ \\
\hline & 1.6 & $2.81 \pm 0.18$ & $347 \pm 20$ & $8.11 \pm 0.67$ & $1.34 \pm 0.13$ \\
\hline & 2.4 & $3.53 \pm 0.23$ & $417 \pm 33$ & $8.52 \pm 0.99$ & $2.02 \pm 0.28$ \\
\hline & 3.2 & $4.19 \pm 0.15$ & $438 \pm 26$ & $9.61 \pm 0.55$ & $2.89 \pm 0.43$ \\
\hline $\operatorname{LSD}(P \leq 0.05)$ & & 0.29 & 34 & 0.90 & 0.28 \\
\hline
\end{tabular}

Table 3

Effects of Si concentration levels on the transpiration rate $(\mathrm{Tr})$, chlorophyll a/b ratio $(\mathrm{Chl} \mathrm{a} / \mathrm{b})$ and carotenoids content of leaves at tuberization stage and mini-tuber potato yield.

\begin{tabular}{|c|c|c|c|c|}
\hline \multirow{3}{*}{$\begin{array}{l}\text { Si concentrations } \\
\left(\mathrm{mmol} \mathrm{L}^{-1}\right)\end{array}$} & \multicolumn{4}{|l|}{ Traits } \\
\hline & $\mathrm{Tr}$ & $\mathrm{Chl} a / b$ & Carotenoids & Mini-tuber yield \\
\hline & $\left(\mathrm{mmol} \mathrm{m} \mathrm{m}^{-2} \mathrm{~s}^{-1}\right)$ & ratio & $\left(\mathrm{mg} \mathrm{g}^{-1} \mathrm{FW}\right)$ & (g per plant) \\
\hline Control & $2.47 \pm 0.39$ & $2.51 \pm 0.14$ & $0.110 \pm 0.007$ & $28.1 \pm 5.8$ \\
\hline 0.8 & $2.22 \pm 0.21$ & $2.53 \pm 0.21$ & $0.112 \pm 0.009$ & $29.4 \pm 5.4$ \\
\hline 1.6 & $2.10 \pm 0.24$ & $2.49 \pm 0.15$ & $0.116 \pm 0.007$ & $30.8 \pm 6.1$ \\
\hline 2.4 & $1.70 \pm 0.17$ & $2.59 \pm 0.18$ & $0.125 \pm 0.013$ & $32.9 \pm 7.1$ \\
\hline 3.2 & $1.53 \pm 0.15$ & $2.76 \pm 0.32$ & $0.115 \pm 0.011$ & $34.6 \pm 7.0$ \\
\hline $\operatorname{LSD}(P \leq 0.05)$ & 0.18 & 0.19 & 0.007 & 1.3 \\
\hline
\end{tabular}


Table 4

Interaction effects of the Si particle size $(\mathrm{P})$ and Si concentration levels $(\mathrm{C})$ on the Chlorophyll a (Chl a), chlorophyll b (Chl b), DPPH radical scavenging and total phenol of potato plantlet leaves at tuberization stage.

\begin{tabular}{|c|c|c|c|c|c|}
\hline \multicolumn{2}{|l|}{ Treatments } & \multicolumn{4}{|l|}{ Traits } \\
\hline C & & Chl a & $\mathrm{Chl} b$ & DPPH radical scavenging & Total phenol \\
\hline & & 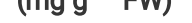 & (mg & (Ascorpate mg & acla $\mathrm{r}$ \\
\hline \multirow{5}{*}{$\begin{array}{l}\text { Nano-Si } \\
\left(\mathrm{mmol} \mathrm{L}^{-1}\right)\end{array}$} & Control & $0.743 \pm 0.066$ & $0.295 \pm 0.018$ & $0.0245 \pm 0.0024$ & $0.458 \pm 0.028$ \\
\hline & 0.8 & $0.776 \pm 0.068$ & $0.308 \pm 0.015$ & $0.0243 \pm 0.0029$ & $0.493 \pm 0.047$ \\
\hline & 1.6 & $0.804 \pm 0.045$ & $0.329 \pm 0.021$ & $0.0267 \pm 0.0038$ & $0.493 \pm 0.050$ \\
\hline & 2.4 & $0.876 \pm 0.077$ & $0.336 \pm 0.030$ & $0.0366 \pm 0.0039$ & $0.624 \pm 0.064$ \\
\hline & 3.2 & $0.952 \pm 0.085$ & $0.346 \pm 0.027$ & $0.0396 \pm 0.0050$ & $0.654 \pm 0.111$ \\
\hline \multirow[t]{5}{*}{ lonized-Si (mmol L-1) } & Control & $0.737 \pm 0.031$ & $0.296 \pm 0.017$ & $0.0243 \pm 0.0017$ & $0.479 \pm 0.040$ \\
\hline & 0.8 & $0.729 \pm 0.041$ & $0.289 \pm 0.015$ & $0.0320 \pm 0.0084$ & $0.471 \pm 0.047$ \\
\hline & 1.6 & $0.758 \pm 0.034$ & $0.301 \pm 0.026$ & $0.0357 \pm 0.0062$ & $0.570 \pm 0.089$ \\
\hline & 2.4 & $0.800 \pm 0.033$ & $0.313 \pm 0.016$ & $0.0257 \pm 0.0021$ & $0.558 \pm 0.063$ \\
\hline & 3.2 & $0.796 \pm 0.039$ & $0.292 \pm 0.017$ & $0.0317 \pm 0.0069$ & $0.543 \pm 0.073$ \\
\hline \multicolumn{2}{|l|}{$\operatorname{LSD}(P \leq 0.05)$} & 0.063 & 0.023 & 0.006 & 0.070 \\
\hline
\end{tabular}

Please insert Tables 2 and 3 near hear

The foliar application of nano and ionized silicon levels imposed different effects on substomatal $\mathrm{CO}_{2}$ concentration. For instance at 2.4 and 3.2 mmol Si $\mathrm{L}^{-1}$, the substomatal $\mathrm{CO}_{2}$ concentration increased significantly compared with control $(P<0.05)$, and the highest amount was observed at 2.4 mmol nano-Si $\mathrm{L}^{-1}$ with a difference of $25.06 \%$ compared with control. The highest effect of ionized-Si particle size was also observed at $3.2 \mathrm{mmol} \mathrm{Si} \mathrm{L}^{-1}$ with an increase of $10.89 \%$ compared to control (Table 2). The application of nano and ionized Si levels changed the mesophyll conductance and the influence of 3.2 mmol ionized-Si $\mathrm{L}^{-1}$, with an increase of $19.97 \%$, was significant compared with control treatment, whereas other ionized-Si levels did not have a significant effect on mesophyll conductance of potato leaves. At 1.6 and $3.2 \mathrm{mmol}$ nano-Si $\mathrm{L}^{-1}$, the mesophyll conductance showed a significant difference of $17.79 \%$ and $33.96 \%$ compared to control, respectively. The highest mesophyll conductance of both Si sizes was observed in $3.2 \mathrm{mmol} \mathrm{Si} \mathrm{L}^{-1}$ (Table 2 ).

Similar to the results of net photosynthetic rate, either ionized or nano Si application improved the water use efficiency of potato and these changes differed under the influence of the particle size. The significant effect of ionized particles on WUE was observed with the increase of $56.59 \%$ and $124.03 \%$ compared with control at 2.4 and $3.2 \mathrm{mmol} \mathrm{Si} \mathrm{L}^{-1}$ levels, respectively. The whole nano-Si levels had a significant effect on the WUE of potato leaves at the tuberization stage and by increasing concentration WUE changes were more noticeable. Furthermore, the influence of nanoparticles was significantly higher than ionized particles at both 1.6 and $2.4 \mathrm{mmol} \mathrm{Si} \mathrm{L}^{-1}$ levels, but this difference was not significant in the highest concentration level (Table 2).

\subsection{Biochemical traits}

The silicon particle size (P) significantly affected chlorophyll $a(\mathrm{Chl} a)$, chlorophyll $b(\mathrm{Chl} b)$, and carotenoids (Cart) content of potato leaves, but no significant effect was observed on chlorophyll $a / b$ ratio $(\mathrm{Chl} a / b)$, the capacity of DPPH radical scavenging and total phenol content. Si concentration levels (C) showed a significant effect on all measured biochemical traits. Although the interaction between $\mathrm{P}$ and $\mathrm{C}$ was not significant on chlorophyll $a / b$ ratio and carotenoids content, these effects were significant in the other biochemical characteristics as well (Table 1).

The application of ionized-Si particles had no significant effect on chlorophyll $a$ and $b$ content, whereas the foliar application of nano-Si levels improved chlorophyll $a$ and $b$ contents in potato leaves. At 2.4 and 3.2 mmol nano-Si $\mathrm{L}^{-1}$ levels, chlorophyll $a$ content was increased by $17.90 \%$ and $28.13 \%$ compared

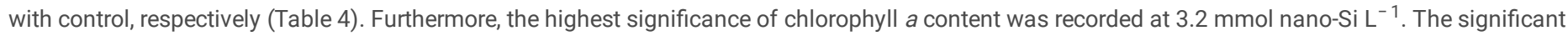
influences of nanoparticles on chlorophyll $b$ content were observed by $11.52,13.90$, and $17.29 \%$ at $1.6,2.4$, and $3.2 \mathrm{mmol} \mathrm{Si} \mathrm{L}^{-1}$ compared with control,

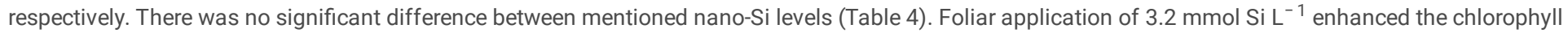
$a / b$ ratio compared with control in potato leaves, whereas the influence of other Si treatments was not significant on this ratio (Table 4). The results showed that the carotenoids content of potato leaves was higher by $6.25 \%$ under nano-Si application than ionized-Si (Table 5). In potato plantlets treated with 2.4 mmol Si L ${ }^{-1}$, carotenoids content was also significantly higher compared with control $(P<0.05)$, whereas other Si treatments was not effective (Table 3 ). 
Table 5

Effects of Si particle size on the carotenoids content of leaves at tuberization stage, mean mini-tuber weight and mini-tuber potato

\begin{tabular}{|llll}
\hline $\begin{array}{l}\text { Si } \\
\text { particle }\end{array}$ & Traits & & \\
\cline { 2 - 4 } & Carotenoids & $\begin{array}{l}\text { Mean } \\
\text { mini- } \\
\text { tuber } \\
\text { weight } \\
\text { (mg g }\end{array}$ & $\begin{array}{l}\text { Mini- } \\
\text { tuber } \\
\text { yield }\end{array}$ \\
\hline Nano & $\begin{array}{l}\text { (g) } \\
\text { (g)er } \\
\text { plant) }\end{array}$ \\
\hline Ionized & $0.119 \pm$ & 7.299 & 32.063 \\
& 0.012 & \pm 1.645 & \pm 6.684 \\
\hline LSD $(P \leq 0.05)$ & $0.112 \pm$ & 7.059 & 30.299 \\
\hline The & 0.008 & \pm 1.977 & \pm 6.447 \\
\hline
\end{tabular}

The presented values are the means \pm standard deviation from thirty data $(n=30)$. LSD: The least significant

difference at $P<0.05$.

Table 6. Correlation analysis of gas exchange parameters, pigment contents, biochemical traits and yield of potato plantlets.

\begin{tabular}{|c|c|c|c|c|c|c|c|c|c|c|c|c|c|}
\hline \multirow[t]{15}{*}{$\begin{array}{l}\text { Nano-Si } \\
\text { treatment }\end{array}$} & Traits & apn & $\operatorname{Tr}$ & $\mathrm{Ci}$ & $\mathrm{MC}$ & Gs & WUE & Chl a & $\mathrm{Chl} b$ & $\begin{array}{l}\mathrm{Chl} \\
a / b\end{array}$ & Cart & DPPH & T.phenol \\
\hline & $\mathrm{Pn}$ & 1 & -0.810 & $0.921^{\star b}$ & $0.934^{\star}$ & 0.123 & $0.958^{*}$ & 0.684 & -0.098 & $0.903^{*}$ & 0.219 & -0.164 & 0.152 \\
\hline & $\operatorname{Tr}$ & $-0.932^{\star}$ & 1 & -0.592 & $-0.913^{*}$ & 0.235 & $-0.937^{*}$ & $-0.932^{\star}$ & -0.278 & $-0.878^{*}$ & -0.703 & -0.155 & -0.687 \\
\hline & $\mathrm{Ci}$ & 0.312 & -0.541 & 1 & 0.722 & 0.054 & 0.785 & 0.523 & -0.074 & 0.673 & -0.029 & -0.449 & -0.126 \\
\hline & $\mathrm{MC}$ & 0.647 & -0.407 & -0.521 & 1 & 0.087 & $0.988^{\star \star}$ & 0.762 & -0.067 & $0.985^{\star \star}$ & 0.395 & 0.109 & 0.412 \\
\hline & Gs & 0.114 & 0.067 & 0.087 & 0.095 & 1 & 0.067 & 0.098 & 0.032 & 0.054 & 0.154 & 0.136 & 0.098 \\
\hline & WUE & $0.986^{\star *}$ & $-0.971^{\star *}$ & 0.462 & 0.515 & 0.214 & 1 & 0.817 & 0.027 & $0.959^{\star *}$ & 0.441 & 0.021 & 0.417 \\
\hline & Chl a & $0.997^{\star \star \star}$ & $-0.939^{*}$ & 0.370 & 0.598 & 0.101 & $0.992^{\star \star *}$ & 1 & -0.200 & 0.673 & 0.858 & -0.086 & 0.773 \\
\hline & Chl $b$ & $0.934^{*}$ & $-0.940^{\star}$ & 0.346 & 0.573 & 0.104 & $0.937^{\star}$ & $0.937^{\star}$ & 1 & -0.200 & 0.815 & -0.352 & 0.654 \\
\hline & $\mathrm{Chl} a / b$ & $0.888^{\star}$ & -0.760 & 0.321 & 0.525 & 0.118 & $0.875^{\star}$ & $0.890^{\star}$ & 0.675 & 1 & 0.308 & 0.246 & 0.362 \\
\hline & Cart & 0.433 & -0.709 & $0.904^{*}$ & -0.325 & 0.064 & 0.570 & 0.475 & 0.570 & 0.256 & 1 & 0.080 & $0.956^{*}$ \\
\hline & DPPH & $0.947^{\star}$ & $-0.920^{*}$ & 0.560 & 0.400 & 0.157 & $0.974^{* *}$ & $0.968^{\star \star}$ & $0.5886^{*}$ & $0.884^{*}$ & 0.589 & 1 & 0.334 \\
\hline & T.phenol & $0.953^{*}$ & $-0.959^{*}$ & 0.580 & 0.388 & 0.194 & $0.987^{\star \star}$ & $0.968^{\star \star}$ & $0.886^{*}$ & $0.887^{\star}$ & 0.634 & $0.987^{\star \star}$ & 1 \\
\hline & TW & -0.705 & -0.822 & 0.191 & 0.495 & 0.102 & 0.712 & 0.682 & 0.835 & 0.362 & 0.554 & -0.560 & 0.633 \\
\hline & TY & $0.982^{\star \star}$ & $-0.962^{\star \star}$ & 0.464 & 0.512 & 0.217 & $0.995^{\star \star \star}$ & $0.992^{\star \star \star}$ & $0.954^{*}$ & $0.850^{\star}$ & 0.575 & $0.980^{\star \star}$ & $0.980^{\star \star}$ \\
\hline
\end{tabular}

a Net photosynthetic rate (Pn), Transpiration rate (Tr), Substomatal $\mathrm{CO}_{2}$ concentration (Ci), Mesophyll conductance (MC), Stomatal conductance (Gs), Water I Chlorophyll $a(\mathrm{Chl} a)$, Chlorophyll $b(\mathrm{Chl} b)$, Carotenoids (Cart), DPPH radical scavenging (DPPH), Total phenol (T. phenol), Mini-tuber weight (TW), Mini-tuber !

$\mathrm{b} * * * *, * * *$ significance level at $P<0.05,0.01$ and 0.001 , respectively.

Please insert Table 4 near hear

Foliar application of nano and ionized Si treatments improved the capacity of leaf DPPH radical scavenging in potato plants, but the influence of particle size

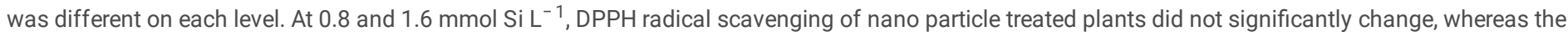
application of ionized showed a significant effect by increasing $31.69 \%$ and $46.91 \%$ compared with control, respectively. However, at 2.4 and 3.2 mmol Si $\mathrm{L}^{-1}$, application of nanoparticles, it was increased by $49.39 \%$ and $61.63 \%$ compared with control, respectively. The influence of nano-Si was also significantly higher than ionized-Si by $42.41 \%$ and $24.92 \%$ at each of 2.4 and $3.2 \mathrm{mmol} \mathrm{Si} \mathrm{L-1}$. Overall, the highest total antioxidant capacity was observed at $3.2 \mathrm{mmol}$ nano-Si L ${ }^{-1}$ (Table 4).

The effect of both particle sizes on total phenol content at $0.8 \mathrm{mmol} \mathrm{Si} \mathrm{L}^{-1}$, were not significant. The highest influence of ionized observed at $1.6 \mathrm{mmol} \mathrm{Si} \mathrm{L}^{-1}$ by $18.99 \%$ difference compared with control but, ionized-Si concentration increased to more than $1.6 \mathrm{mmol} \mathrm{Si} \mathrm{L}^{-1}$, phenol content decreased (Table 4 ). Increasing concentration of nano-Si particles also improved the total phenol content of potato leaves and the highest total phenol content was observed at 2.4 and $3.2 \mathrm{mmol}$ nano-Si L ${ }^{-1}$ by $36.24 \%$ and $42.79 \%$ difference compared with control, respectively (Table 4 ). The difference between 2.4 and $3.2 \mathrm{mmol}$ nano-Si $\mathrm{L}^{-1}$ levels was also not significant. The total phenol content of nanoparticle treated plants was significantly higher than ionized at $3.2 \mathrm{Si}$ mmol $\mathrm{L}^{-1}$ (Table 4 ). 


\subsection{Mini-tuber yield}

The silicon particle size (P) was significantly effective on the mean of mini-tuber weight (TW) and mini-tuber yield (TY) (Table 1). Silicon concentration levels (C) also showed a significant effect on mini-tuber yield, but their sole and interaction effects were not significant on the mean mini-tuber weight. In comparison with the ionized-Si treatment, the nano-Si significantly increased the mean of mini-tuber weight (Table 5), and a similar result was observed in the mini-tuber yield (Table 5). The potato mini-tuber yield significantly improved by increasing the Si concentration levels, and all Si treatments showed a significant difference with control $(P<0.05)$. These changes increased by increasing Si levels and the highest mini-tuber yield was observed at $3.2 \mathrm{mmol} \mathrm{Si} \mathrm{L}^{-}$ 1 (Table 3).

\section{Please insert Table 5 near hear}

\subsection{Correlation results}

The correlations among the different characteristics were calculated using Pearson's correlation coefficients (Table 6). As shown in Table 6, mini-tuber yield was positively and significantly correlated with $\mathrm{Pn}(P<0.01)$, WUE $(P<0.001)$, Chl a $(P<0.001), \mathrm{Chl} \mathrm{b}(P<0.05), \mathrm{Chl}$ a/b ratio $(P<0.05)$, scavenging DPPH radical $(P<0.01)$ and total phenol $(P<0.01)$ in nano-Si treatments and negative significant relationship was observed between mini-tuber yield and $\operatorname{Tr}(P<$ 0.01). In ionized-Si treatment, mini-tuber yield was positively and significantly correlated with WUE $(P<0.05)$ and Chl a $(P<0.05)$. The Tr in both nano-Si and ionized-Si treatments showed negative relationship with other measuring characteristics.

Please insert table 6 near hear

\section{Discussion}

In this experiment, the foliar application of silicon concentrations enhanced gas exchange parameters, pigment contents, antioxidant capacity, and mini-tuber yield of potato which are consistent with previous reports regarding the beneficial influences of Si supplementation on antioxidant activities, growth and yield of other crops including cucumber [5], barley [27], soybean [29, 30], banana [14] and tomato [8].

In this study, the increasing Pn and pigment content was apparent at concentrations above $1.6 \mathrm{mmol} \mathrm{L}^{-1}$ of both Si sizes. These results were in agreement with studies on tomato, cucumber, and soybean which indicated the positive influence of Si supplement on the Pn and Chl content of leaves in hydroponic conditions $[5,8,30]$. Furthermore, our results indicated that DPPH radical scavenging was clearly increased by Si particles in the tuberization stage of potato. There might be numerous mechanisms involved in the influence of silicon on net photosynthesis however, an increase in antioxidant capacity as a result of Si induction may be the possible mechanism. Feng et al. (2010) [10] showed that Si could promote net photosynthesis, which is related to the individual role of $\mathrm{Si}$ in protecting photosynthesis apparatus from ROS damages. Furthermore, in similar previous reports it was proposed that silicon helps to improve the stability of cell plasma membranes [29], the integrity (thylakoids, grana lamellae) and function of chloroplast [8, 9, 10] and following that the electron transport chain in thylakoid membranes for the production of ATP and NADPH will be protected against ROS [9] by promoting antioxidant system for detoxifying reactive oxygen species $[10,30]$. Our results also showed that total phenol content in potato leaves was enhanced significantly by Si at above $0.8 \mathrm{mmol}^{-1}$ levels. These observations are in agreement with the findings of Gagoonani et al. (2011) [17], who applied 1.5 mM Si which improved phenolic components of leaves at the control and Al toxicity treatments in Brago officinalis L. seedling was grown in hydroponically medium. Moreover, Shetty et al. (2011) [18] reported that the application of $3.6 \mathrm{mM}$ Si stimulated phenolic acids and flavonoids in rose (Rosa hybrida). They suggested that Si can promote the expansion of genes encoding enzymes and transcript levels in the phenylpropanoid pathways compared with untreated Si plants. Phenolic components can impress plant development via lignin and pigment biosynthesis or accumulation in the subepidermal layers of plant tissues [30] and the up-regulation biosynthesis of phenols in chloroplasts could enhance radiation intercept in leaves [31]. So, increasing phenol content induced by Si has influenced the structure, function, and protection system of potato leaves, especially in the chloroplast. Since increase of DPPH radical scavenging and total phenols content under the influence of silicon was simultaneously accompanied with improvement in photosynthetic pigments, the probable conclusion can be that partial increasing concentration of photosynthesis pigments (chlorophyll $a$ and $b$ ) may depend on maintaining ultrastructure and orderliness of chloroplast or improving of chlorophyll biosynthetic pathways as a consequence of Si-related up-regulation of antioxidant system and phenol components.

Several previous reports have shown silicon can regulate the activities of main photosynthetic enzymes of Calvin cycle [5, 11]. Adatia and Bestford (1986) [5] reported that the Si addition to nutrient solution enhanced carboxylase activity (RubisCO) of cucumber leaves under normal conditions. Similar findings were reported for barley [27] and Spartina densiflora [11] under saline and Cu toxicity stresses. Silicon application increased the activity of phosphoenol pyruvate carboxylase in wheat under drought conditions [32]. Hence it seems that in this experiment, part of the Si influence has been linked with activity regulation of key enzymes in non-photochemical photosynthetic processes. Moreover, up-regulation endogenous phytohormones such as GAs, IAA, and cytokines in Sitreated plants as reported for mango under drought stress [33] or $\mathrm{GA}_{1}$ in soybean leaves under normal hydroponically conditions [28] mentioned in previous studies. In conclusion, it is likely that enhance of chlorophyll content and Pn were correlated with an increase of growth regulators in Si-treated potato leaves.

Results of this study indicated that by increasing Si concentration, Chl $b$ content was significantly increased at 1.6 and carotenoids increased at 3.2 mmol $\mathrm{L}^{-1}$ of Si. The $\mathrm{Chl} b$ and carotenoids are considered as an antenna and auxiliary pigments for $\mathrm{Chl}$ a reaction centers. Therefore, an increase in carotenoids and $\mathrm{Chl}$ $b$ can be helpful for the absorption of light energy for electron transport photosystems in Chl $a$ [34]. It is possible to suggest a positive role of Si in controlling photoinhibition of potato leaves. Since the absorption spectrum of $\mathrm{Chl} a$ and $\mathrm{Chl} b$ are different, it seems that an increase in the Chl $a / b$ ratio can determine the quality of light-harvesting by leaf. Moreover, the finding of Kitajima and Hogan (2003) verified that improvement of Chl $a / b$ accompanied by an increase in the electron transport rate in reaction centers of $\mathrm{Chl} a$ and rubisco carboxylation capacity which are in agreement with our results that shown a positive correlation between $\mathrm{Pn}$ with $\mathrm{Chl} a$ and $\mathrm{Chl} a / b$ in two scales of Si particle treatments (Table 6). 
Based on this study results, an increase of Pn was simultaneously accompanied with decrease of Tr in Si-treated leaves at the tuberization growth stage. Nevertheless, the Gs was not affected by Si treatments (Tables 1 and 2). If the limitation of $\operatorname{Tr}$ was due to stomatal closure, there should be a decrease in Ci. However, Ci had a slight increase in Si-treated leaves with 2.4 and $3.2 \mathrm{mmol} \mathrm{L}^{-1}$. Therefore, the decrease in Tr may be due to nonstomatal restrictions which are in agreement with the results reported for $S$. densiflora [11] (Mateos-Naranjo et al. 2015) and tomato [35] treated with Si under salinity stress in greenhouse conditions. Various studies have described that by applying of silicon, the silica cuticle double layer was formed on the leaf epidermis which may reduce water loss through the cuticles of plants $[11,14,36]$. Although, the cuticular transpiration rate is lower than the stomatal transpiration rate, it can perform an important role in leaf water loss. Therefore, it seems that the decrease of $\mathrm{Tr}$ in Si-treated potato leaves most likely has been due to the reduction of cuticular Tr. Asmar et al. (2013) [14] suggested that the Si accumulation in epidermal tissue can have a positive effect on water relations in leaves during acclimatization under humidity changes in greenhouse conditions. Moreover, physical strength caused by Si deposition may develop mechanical protection to infection pathogens in crop leaves [18]. Therefore, it seems that foliar application of Si particles can be useful for the growth and health of potato plantlets are transferred to soilless culture. Our results also indicated that stomatal conductance did not change (Table 1 ) and it has not limited the $\mathrm{CO}_{2}$ diffusion into substomata chamber or $\mathrm{CO}_{2}$ assimilation in chloroplasts. Accordingly, an increase of $\mathrm{Pn}$ with Si may associate with the photosynthetic enzymatic process and chloroplast function.

Overall, silicon particles levels improved water use efficiency. Previous studies are in agreement with these results indicated that Si can enhance water use efficiency at the normal conditions in tomato $[8,36]$. Our results showed that a decrease in transpiration rate was accompanied by an increase of Pn in Sitreated leaves. Therefore, improvement of water use efficiency was predictable. A positive effect of Si application on mesophyll conductance of potato leaves was also in agreement with Haghighi and Pessarakli (2013) [8] results in cherry tomato. Our results showed that all Si levels had a positive effect on minituber yield. The ability of Si to increase yield production has been demonstrated in cucumber [5] and tomato [35].

According to this study, although net photosynthesis, pigment content, and yield measured in Si treatments were enhanced. These changes in nano-Si treated plants were more than ionized-Si. These results also indicated that potato leaves could foliar uptake of Si and nanoscale particles showed more efficiency in response to this method. The higher influence of nanoparticles may be due to unique characteristics $[8,12,37]$ and facility uptake by leaf stomata because of their smaller size. These observations are in agreement with Tripathi et al. (2015) [12], who achieved the beneficial influence of nano-Si on growth and dry weight of Pisum sativum in normal and Cr toxicity. Siddiqui and Al-Whaibi (2014) [36] also confirmed that nano-Si increased the germination characteristics, which enhanced the seedling dry weight of tomato. However, Haghighi and Pessarakli (2013) [8] showed that although Si addition mitigated adverse effects of salinity in gas exchange parameters and dry weight of cherry tomato, no difference between root application of nano and bulk Si was observed. The comparison of silicon nanoparticles and silicate in Fenugreek by Nazaralian et al. (2017) [6] also indicated that the influence of the added nanoparticles in nutrient solution declined over time. Moreover, the results of Abdel-Haliem et al. (2017) [37] showed that application of nano and ions Si in rice seedling under saline conditions increased growth, antioxidant activity, and biochemical traits such as soluble carbohydrates and amino acids, but the difference between particle sizes of Si was not noticeable.

Stomatal uptake can be a main pathway for the foliar uptake of mineral nutrients and other solutes [34] (Taiz et al., 2015), which can not able to penetrate through the surface of the epidermal cells. There are very fine pores with a diameter on a nanoscale on adaxial and abaxial leaf surface which are called ectodesmota with an approximate density of $10^{10}$ per $\mathrm{cm}^{-2}$ leaf area. Moreover, inside of this pores are covered with polygalacturonic acids, which only allow positive particles to enter [38] (Marschner 1995). It can be concluded that since in our study silicon solutions were acidic ( $\mathrm{pH}=5)$ and ectodesmota diameter was nanoscale, it seems that the high influence of nano-Si treatments may be due to an increase of their foliar uptake via ectodesmota in potato leaves.

\section{Conclusion}

In this study, foliar application of Si particles imposed a remarkably positive role in the improving of photosynthetic, biochemical characteristics, and minituber yield of potato. Moreover, the nanoparticles was more effective than ionized-Si in many measured traits. As a result, it could be recommended that $\mathrm{Si}$ application improve the safety and tuber propagation of potato plantlets in soilless culture conditions. To maximize the influence of Si treatment, the use of nanoparticles will be a proper strategy.

\section{Declarations}

\section{Declaration of interests}

$\otimes$ The authors declare that they have no known competing financial interests or personal relationships that could have appeared to influence the work reported in this paper.

$\bigotimes$ The authors declare the following financial interests/personal relationships which may be considered as potential competing interests:

Acknowledgements Authors are thankful to the Ferdowsi University of Mashhad (FUM) for financial support to carry out this work.

Funding This study was financed in part by Ferdowsi University of Mashhad (FUM).

\section{Author contribution}

Bijan Saadatian designed and performed the experiments, Mohamad Kafi was involved in planning and supervised the project, Mohamad Banayan aval conceived the original idea and supervised the project Hossein Hammami Processed the experimental data, performed the analysis, wrote the manuscript with support from all authors. All authors discussed the results and contributed to the final manuscript. 
Data Availability Not applicable.

\section{Compliance with Ethical Standards}

Conflicts of Interest None.

Ethics Approval Not applicable.

Consent to Participate Not applicable.

Consent for Publication Not applicable.

Code Availability Not applicable.

Research involving Human Participants and/or Animals Not applicable.

\section{References}

1. FAO (2019) Website of the FAO. Available online at http://faostat.fao.org

2. Khawla BJ, Sameh M, Imen G, Donyes F, Dhouha G, Raoudha EG, Oumèma NE (2014) Potato peel as feedstock for bioethanol production: A comparison of acidic and enzymatic hydrolysis. Ind Crops Prod 52:144-149

3. Ma X, Wang Y, Liu M, Xu J, Xu Z (2015) Effects of green and red lights on the growth and morphogenesis of potato (Solanum tuberosum L.) plantlets in vitro. Sci Hortic 190:104-109

4. Ritter E, Angulo B, Riga P, Herran C, Relloso J, San Jose M (2001) Comparison of hydroponic and aeroponic cultivation systems for the production of potato minitubers. Potato Res 44:127-135

5. Adatia MH, Bestford RT (1986) The effect of silicon in cucumber plants grown in recirculating nutrient solution. Ann Bot 58:343-351

6. Nazaralian S, Majd A, Irian S, Najafi F, Ghahremaninejad F, Landberg T, Greger M (2017) Comparison of silicon nanoparticles and silicate treatments in fenugreek. Plant Physiol Biochem 115:25-33

7. Rastogi A, Tripathi DK, Yadav S, Chauhan DK, Živčák M, Ghorbanpour M, Ibrahim ElSheery N, Brestic M (2019) Application of silicon nanoparticles in agriculture. Biotech 9:90

8. Haghighi M, Pessarakli M (2013) Influence of silicon and nano-silicon on salinity tolerance of cherry tomatoes (Solanum lycopersicum L.) at early growth stage. Sci Hortic 161:111-117

9. Cao B, Ma Q, Zhao Q, Wang L, Xu K (2015) Effects of silicon on absorbed light allocation, antioxidant enzymes and ultrastructure of chloroplasts in tomato leaves under simulated drought stress. Sci Hortic 194:53-62

10. Feng J, Shi Q, Wang X, Wei M, Yang F, Xu H (2010) Silicon supplementation ameliorated the inhibition of photosynthesis and nitrate metabolism by cadmium (Cd) toxicity in Cucumis sativus L. Sci Hortic 123:521-530

11. Mateos-Naranjo E, Gallé A, Florez-Sarasa I, Alejandro Perdomo J, Galmés J, Ribas-Carbó M, Flexas J (2015) Assessment of the role of silicon in the Cutolerance of the $\mathrm{C}_{4}$ grass Spartina densiflora. J Plant Physiol 178:74-83

12. Tripathi DK, Singh VP, Prasad SM, Chauhan DK, Dubey NK (2015) Silicon nanoparticles (SiNp) alleviate chromium (VI) phytotoxicity in Pisum sativum (L.) seedlings. Plant Physiol Biochem 96:189-198

13. Epstein E (2009) Silicon: its manifold roles in plants. Ann Appl Biol 155:155-160

14. Asmar A, Castro EM, Pasqual M, Pereira FJ, Soares JDR (2013) Changes in leaf anatomy and photosynthesis of ionizedpropagated banana plantlets under different silicon sources. Sci Hortic 161:328-332

15. Sattar A, Cheema MA, Ali H, Sher A, ljaz M, Hussain M, Hassan W, Abbas T (2016) Silicon mediates the changes in water relations, photosynthetic pigments, enzymatic antioxidants activity and nutrient uptake in maize seedling under salt stress. Grassl Sci 62:262-269

16. Wang S, Wang F, Gao S (2014) Foliar application with nano-silicon alleviates Cd toxicity in rice seedlings. Environ Sci Pollut Res 22:2837-2845

17. Gagoonani S, Enteshari S, Delavar K, Behyar M (2011) Interactive effects of silicon and aluminum on the malondialdehyde (MDA), proline, protein and phenolic compounds in Borago officinalis L. Journal of Medicinal Plants Research 5:5818-5827

18. Shetty R, Frette X, Jensen B, shety PN, Jensen JD, Jørgensen HJL, Newman M, Christensen LP (2011) Silicon-induced changes in antifungal phenolic Acids, flavonoids, and Key phenylpropanoid pathway genes during the interaction between miniature Roses and the biotrophic pathogen Podosphaera pannosa. Plant Physiol 157:2194-2205

19. Zhang M, Liang Y, Chu G (2017) Applying silicate fertilizer increases both yield and quality of table grape (Vitis vinifera L.) grown on calcareous grey desert soil. Sci Hortic 225:757-763

20. Navarro E, Baun A, Behra R, Hartmann NB, Filser J, Miao AJ, Quigg A, Santschi PH, Sigg L (2008) Environmental behavior and ecotoxicity of engineered nanoparticles to algae, plants, and fungi. Ecotoxicology 17:372-386

21. Haghighi M, Afifipour Z, Mozafarian M (2012) The effect of N-Si on tomato seed germination under salinity levels. Journal of Biodiversity Environmental Science 6:87-90

22. Mahdavi S, Kafi M, Fallahi E, Shokrpour M, Tabrizi L (2016) Water stress, nano silica, and digoxin effects on minerals, chlorophyll index, and growth of ryegrass. International Journal of Plant Production 10:251-264

Page $10 / 11$ 
23. Hoagland DR, Arnon DI (1950) The water-culture method for growing plants without soil. Circular California Agricultural Experiment Station 347:1-32

24. Arnon DI (1949) Copper enzymes in isolated chloroplasts. Polyphenoloxidase in Beta vulgaris. Plant Physiol 24:1-15

25. Singleton VL, Rossi JA (1965) Colorimetry of Total Phenolics with Phosphomolybdic-Phosphotungstic Acid Reagents. Am J Enol Vitic 16:144-158

26. Abe N, Murata T, Hirota A (1998) Novel 1,1-diphenyl-2-picryhy-drazyl- radical scavengers, bisorbicillin and demethyltrichodimerol, from a fungus. Bioscience biotechnology biochemistry 62:661-662

27. Liang YC (1998) Effect of Si on leaf ultrastructure, chlorophyll content and photosynthetic activity in barley under salt stress. Pedosphere 8:289-296

28. Lee SK, Sohn EY, Hamayun M, Yoon JY, Lee IJ (2010) Effect of silicon on growth and salinity stress of soybean plant grown under hydroponic system. Agrofor Syst 80:333-340

29. Shen X, Zhou Y, Duan L, Li Z, Eneji AE, Li J (2010) Silicon effects on photosynthesis and antioxidant parameters of soybean seedlings under drought and ultraviolet-B radiation. J Plant Physiol 167:1248-1252

30. Clé C, Hill LM, Niggeweg R, Martin CR, Guisez Y, Prinsen E, Jansen MAK (2008) Modulation of chlorogenic acid biosynthesis in Solanum lycopersicum; consequences for phenolic accumulation and UV-tolerance. Phytochemistry 69:2149-2156

31. Kefeli VI, Kalevitch MV, Borsari B (2003) Phenolic cycle in plants and environment. Journal of Cell Molecular Biology 2:13-18

32. Gong HJ, Chen KM (2012) The regulatory role of silicon on water relations, photosynthetic gas exchange, and carboxylation activities of wheat leaves in field drought conditions. Acta Physiol Plant 34:1589-1594

33. Helaly MN, El-Hoseiny H, El-Sheery NI, Rastogi A, Kalaji HM (2017) Regulation and physiological role of silicon in alleviating drought stress of mango. Plant Physiol Biochem 118:31-44

34. Taiz L, Zeiger E, Møller IM, Murphy A (2015) Plant physiology and development. sixth ed. Published by Sinauer associates

35. Romero-Aranda MR, Jurado O, Cuartero J (2006) Silicon alleviates the deleterious salt effect on tomato plant growth by improving plant water status. J Plant Physiol 163:847-855

36. Siddiqui MH, Al-Whaibi MH (2014) Role of nano-SiO 2 in germination of tomato (Lycopersicum esculentum seeds Mill.). Saudi Journal of Biological Sciences 21:13-17

37. Abdel-Haliem MEF, Hegazy HS, Hassan NS, Nagui DM (2017) Effect of silica ions and nano silica on rice plants under salinity stress. Ecol Eng 99:282289

38. Marschner H (1995) Mineral Nutrition of Higher Plants. Academic Press, London, p 887

\section{Figures}

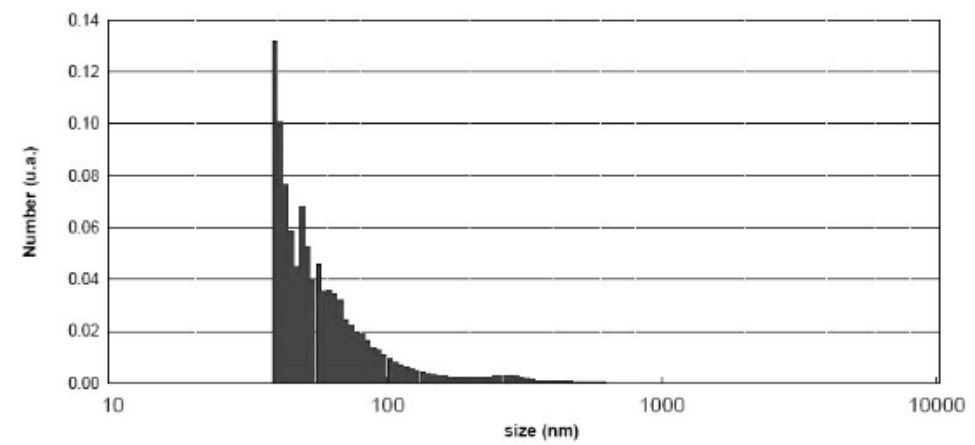

\section{Figure 1}

The result of particle size analyzer (PSA) nano Si particles.

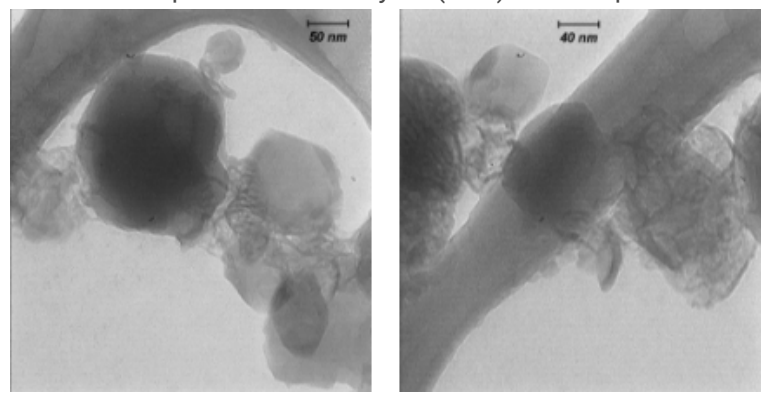

\section{Figure 2}

Scanning Electron lonizedscope (SEM) ionizedgraph of nano-Si particles with magnification of 40000X (a) and 50000X. 